\title{
PAPER
}

\section{Outcome of contemporary surgery for chronic subdural haematoma: evidence based review}

\section{R Weigel, P Schmiedek, J K Krauss}

See Editorial Commentary, p 842

J Neurol Neurosurg Psychiatry 2003;74:937-943

See end of article for authors' affiliations

.....................

Correspondence to: Prof Dr Joachim K Krauss, Department of Neurosurgery, University Hospital, Klinikum Mannheim, Theodor Kutzer Ufer 1-3, D-68167 Mannheim, Germany; joachim.krauss@ nch.ma.uni-heidelberg.de

Received 9 July 2002

In revised form

8 January 2003

Accepted 4 March 2003
Objective: To evaluate the results of surgical treatment options for chronic subdural haematoma in contemporary neurosurgery according to evidence based criteria.

Methods: A review based on a Medline search from 1981 to October 2001 using the phrases "subdural haematoma" and "subdural haematoma AND chronic". Articles selected for evaluation had at least 10 patients and less than $10 \%$ of patients were lost to follow up. The articles were classified by three classes of evidence according to criteria of the American Academy of Neurology. Strength of recommendation for different treatment options was derived from the resulting degrees of certainty.

Results: 48 publications were reviewed. There was no article that provided class I evidence. Six articles met criteria for class II evidence and the remainder provided class III evidence. Evaluation of the results showed that twist drill and burr hole craniostomy are safer than craniotomy; burr hole craniostomy and craniotomy are the most effective procedures; and burr hole craniostomy has the best cure to complication ratio (type $\mathrm{C}$ recommendation). Irrigation lowers the risk of recurrence in twist drill craniostomy and does not increase the risk of infection (type $\mathrm{C}$ recommendation). Drainage reduces the risk of recurrence in burr hole craniostomy, and a frontal position of the drain reduces the risk of recurrence (type $B$ recommendation). Drainage reduces the risk of recurrence in twist drill craniostomy, and the use of a drain does not increase the risk of infection (type $C$ recommendation). Burr hole craniostomy appears to be more effective in treating recurrent haematomas than twist drill craniostomy, and craniotomy should be considered the treatment of last choice for recurrences (type $\mathrm{C}$ recommendation). Conclusions: The three principal techniques-twist drill craniostomy, burr hole craniostomy, and craniotomy-used in contemporary neurosurgery for chronic subdural haematoma have different profiles for morbidity, mortality, recurrence rate, and cure rate. Twist drill and burr hole craniostomy can be considered first tier treatment, while craniotomy may be used as second tier treatment. A cumulative summary of data shows that, overall, the postoperative outcome of chronic subdural haematoma has not improved substantially over the past 20 years.
C hronic subdural haematoma of the elderly is nowadays often considered to be a rather benign entity, ignoring its relatively high mortality and morbidity. When Virchow first described "pachymeningitis haemorrhagica interna," however, it was considered a fatal disorder. ${ }^{1}$ Over the past 150 years, a dramatic improvement in outcome was achieved following better understanding of the pathophysiology, the introduction of modern imaging methods, and refinement of operative techniques. ${ }^{2}$ However, mortality of up to $13 \%$ is still reported in contemporary literature, ${ }^{3-6}$ which may reflect the fact that up to four deaths a year are related to this condition in a typical neurosurgical department. Although this is one of the most frequent problems encountered in neurosurgery, there has been relatively little progress in its treatment during the past 20 years. This is in marked contrast to the development of sophisticated concepts and surgical techniques in other subspecialties of neurosurgery, such as functional, spinal, or vascular neurosurgery.

The principal techniques used in the treatment of chronic subdural haematoma are presently twist drill craniostomy, burr hole craniostomy, and craniotomy. Other procedures are undertaken much more rarely. Additional procedures include intraoperative irrigation of the subdural space and drainage of the haematoma. The number of treatment options reflects the dilemma of the search for the optimum procedure.

In this paper, we provide the first evidence based review of the contemporary surgical treatment of chronic subdural haematoma.

\section{METHODS}

A systematic review was undertaken by conducting a Medline search from January 1981 to October 2001. The key words "subdural haematoma" and "subdural haematoma AND chronic" yielded a total of 973 publications. Additionally, reference lists of recent publications cited another 198 papers on the topic. In order to reduce possible publication bias and enhance the quality of the analysis, certain inclusion criteria were set arbitrarily, in agreement with similar analyses on other topics. To exclude inherent positive bias from case reports and small series, only articles reporting on 10 patients or more were selected for analysis. Paediatric series or mixed series without separate statistical analysis for subgroups were not evaluated. As mortality and morbidity rates might be falsely low in series with high attrition rates, only studies with less than $10 \%$ of patients lost to follow up at the time of statistical evaluation were included. Openings of the skull up to a diameter of $5 \mathrm{~mm}$ were categorised as twist drill craniostomy, openings of up to $30 \mathrm{~mm}$ as burr hole craniostomy or enlarged burr hole craniostomy, and larger openings as craniotomy.

Reports written in English or German were considered, but reports written in other languages were excluded.

Each article was classified as providing class I, class II, or class III evidence according to the criteria of the American Academy of Neurology (AAN), ${ }^{7}$ which are similar to those of the American Association of Neurological Surgeons (AANS). ${ }^{8}$ The strength of the recommendations for management are derived from the resulting degrees of certainty (table 1).

In order to allow both comparison and analysis of the summarised data from different studies, uniform criteria were 
Table 1 Overview of evidence based criteria

Classes of evidence

Class I: Evidence provided by one or more well designed randomised controlled clinical studies.

Class II: Evidence provided by one or more well designed clinical studies such as prospective open, case-control studies, etc.

Class III: Evidence provided by expert opinion, non-randomised historical controls, or case reports of one or more patients.

Strength of recommendations

Type A: Strong recommendation, based on class I evidence or overwhelming class II evidence when circumstances preclude randomised clinical trials.

Type B: Recommendation based on class II evidence.

Type C: Recommendation based on strong consensus of class III evidence.

Classes of evidences and strength of recommendations adopted from the guidelines of the American Academy of Neurology?

Table 2 Overview of contemporary neurosurgical treatment options for chronic subdural haematoma of the elderly

\begin{tabular}{|c|c|c|c|}
\hline \multirow{2}{*}{$\begin{array}{l}\text { Procedure } \\
\text { Craniotomy }\end{array}$} & \multicolumn{2}{|c|}{ Supplementary procedures } & \multirow{2}{*}{$\begin{array}{l}\text { No of studies (refs) } \\
6(19,21,24,38,50,52)\end{array}$} \\
\hline & Irrigation & Drainage & \\
\hline Craniotomy & No irrigation & No drainage & $4(21,39,43,45)$ \\
\hline Burr hole & No irrigation & No drainage & $2(21,37)$ \\
\hline Burr hole & Irrigation & No drainage & $7(15,25,31,36,43,51,55)$ \\
\hline Burr hole & No irrigation & Drainage & $4(14,21,45,56)$ \\
\hline Burr hole & Irrigation & Drainage & $\begin{array}{l}28(3,4,9,17-20,22,24,27,28, \\
30,32,33,35,38,40,42,46-54, \\
56)\end{array}$ \\
\hline Burr hole & \multicolumn{2}{|c|}{$\begin{array}{l}\text { Drainage, continuous inflow and outflow } \\
\text { irrigation }\end{array}$} & $2(24,40)$ \\
\hline Twist drill & No irrigation & No drainage & $2(12,41)$ \\
\hline Twist drill & No irrigation & Drainage & $4(6,16,44,46)$ \\
\hline Burr hole $+\mathrm{CO}_{2}$ & & & $1(26)$ \\
\hline Twist drill $+\mathrm{O}_{2}$ & & & $1(13)$ \\
\hline Endoscopy & & & $1(23)$ \\
\hline Subduro-peritoneal shunt & & & $1(39)$ \\
\hline
\end{tabular}

used to define morbidity, mortality, and recurrence. Morbidity was defined as any complication during or after surgery other than recurrence. Mortality included any death reported between surgery and discharge from hospital. Recurrence was defined, in general, by clinical and radiological findings. Morbidity and mortality were determined at the time of discharge and at the time of last follow up. For comparison (figs 1-3), only those studies were analysed that provided detailed information on morbidity and mortality, or provided the raw data on these outcomes. We considered morbidity and mortality to be a measure of the safety of a procedure. The cure rate indicated the percentage of patients who reached full autonomy after surgery (grade 0 or grade 1 in the Markwalder ${ }^{9}$ or Bender ${ }^{10}$ classifications, or grade 5 on the Glasgow outcome scale $\left.^{11}\right)$. The cure rate therefore reflects the efficiency of a particular surgical method. The recurrence rate was considered to be a reciprocal measure of effectiveness in treating the underlying cause of the disease.

\section{Statistics}

For statistical analysis we used Sigma Stat, version 2.03 (SPSS Inc). Patient data of corresponding treatment groups from different publications were summarised and statistically compared using the $\chi^{2}$ test. In all circumstances a probability (p) value of $<0.05$ was considered significant.

\section{RESULTS}

Medline search and review of reference lists yielded 48 articles that were suitable for evaluation. ${ }^{346912-55}$ None of the articles reviewed met the criteria for class I evidence. Six articles, ${ }^{4} 930313540$ of which four ${ }^{403135}$ were concerned with the question of whether drainage systems should be used, met criteria for class II evidence. The majority of publications met criteria for class III evidence. On the basis of this classification and weighting according to evidence based criteria, the following summarising statements concerning surgical approach, irrigation, drainage, and treatment of recurrences were made.

\section{Evidentiary summary of results \\ Surgical approach}

Surgical approaches are restricted to the three principal techniques with very few exceptions: twist drill craniostomy $^{6} 1213164144$ 46; burr hole craniostomy ${ }^{3} 491415$ 17-22 30-33 35-38 4042 43 45-56; and craniotomy. ${ }^{19} 2124383943455052$

Combining each approach with the use of intraoperative irrigation or the use of drainage provides a variety of treatment options (table 2). Few other technical variants were described. Aoki reported filling the subdural space with 100\% oxygen after haematoma evacuation, ${ }^{13}$ while Kitakami and colleagues used carbon dioxide. ${ }^{26}$ Recurrent chronic subdural haematoma was successfully treated endoscopically through a small burr hole by Hellwig et al. ${ }^{23}$ A different approach was presented by Probst, who demonstrated the benefit of subduro-peritoneal shunting of chronic subdural haematomas in the elderly. ${ }^{39}$

Overall, there was no significant difference in mortality between the three techniques. Figure 1 summarises mortality, morbidity, and cure and recurrence rates for the three principal techniques. Morbidity was significantly higher in the craniotomy series $(12.3 \%)$ than with twist drill craniostomy $(3 \%)$ or burr hole craniostomy $(3.8 \%)$. Differences in cure rates did not reach statistical significance. Both burr hole craniostomy and craniotomy had lower recurrence rates than twist drill craniostomy $(\mathrm{p}<0.001)$. 

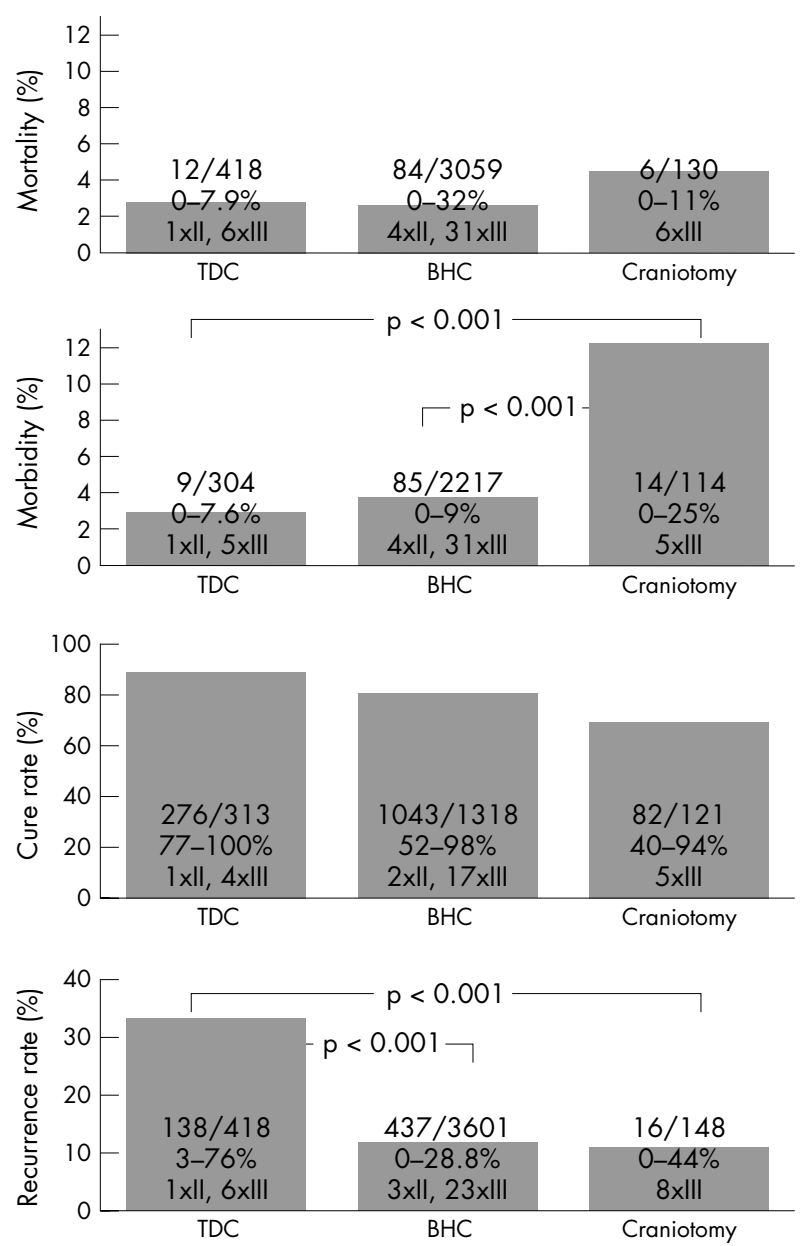

Figure 1 Comparison between the three principal surgical techniques for treatment of chronic subdural haematoma. Mortality, morbidity, cure rate, and recurrence rate are compared for twist drill craniostomy (TDC), burr hole craniostomy $(\mathrm{BHC})$, and craniotomy. The grey columns show the relative percentage of summarised data on the corresponding treatment groups from different publications. The legends in the columns show absolute numbers, the range of relative values, and the number of studies which provided statistical data, with their classes of evidence.

Two of nine class III evidence publications on craniotomy 192124383943455052 reported comparison with another principal technique. Schulz and colleagues ${ }^{45}$ evaluated the results of burr hole craniostomy $(\mathrm{n}=30) \quad v$ craniotomy $(\mathrm{n}=35)$. They found markedly fewer complications in the burr hole craniostomy group (3\%) than in the craniotomy group (34\%), while mortality and recurrence rates did not differ significantly. Hamilton and colleagues ${ }^{21}$ compared four different treatment options within the framework of a retrospective analysis. The four groups included burr hole craniostomy with drainage $(n=14)$ and without drainage $(n=29)$, and craniotomy with drainage $(n=29)$ and without drainage $(n=20)$. Results were comparable for all four groups.

Only one of seven publications $s^{612131641446}$ compared twist drill craniostomy with another principal technique. Smely et al compared a prospective series of chronic subdural haematoma patients $(\mathrm{n}=33)$ undergoing twist drill craniostomy with a historical control series of burr hole craniostomies $(\mathrm{n}=33){ }^{46}$ Twist drill craniostomy significantly surpassed the results of the burr hole technique in lowering morbidity ( $0 \% \vee 18 \%)$, recurrence rate ( $18 \% v 39 \%)$, and duration of hospital stay (4.9 $v 9.6$ days). The recurrence rate of $39 \%$ in the burr hole group, however, is the highest in all published reports. The recurrence rate of $18 \%$ in the twist drill group is also above average for burr hole craniostomy series $(12.1 \%)$ or craniotomy series $(10.8 \%)$

\section{Irrigation}

Three class III evidence publications provide data allowing direct comparison of burr hole craniostomy with and without intraoperative irrigation. Morbidity, mortality, and cure rates were similar between groups in each publication. Suzuki and associates reported a recurrence rate of $3.4 \%$ for the group without irrigation, and 3\% for the group with irrigation. The difference did not reach significance. ${ }^{49}$ In the series by Matsumoto et al eight of 121 patients had no intraoperative irrigation. There were no significant differences in the recurrence rates. ${ }^{32}$ In Kuroki's series, the recurrence rate was $3.6 \%$ without irrigation and $13.3 \%$ with irrigation. ${ }^{56}$ This difference did not, however, reach significance.

Two publications report on the use of continuous inflow and outflow irrigation after surgical decompression of chronic subdural haematomas. Ram et al found fewer recurrences in their postoperative irrigation group than in their control group $(1 / 19 v 4 / 18)$ (class II evidence).$^{40}$ Owing to the small number of recurrences, however, the difference did not reach significance. Similar results were reported by Hennig and Kloster, $^{24}$ who retrospectively compared four different variables (class III evidence): burr hole craniostomy with continuous inflow and outflow drainage (group 1); burr hole craniostomy with intraoperative irrigation and postoperative closed system drainage (group 2); burr hole craniostomy with intraoperative irrigation only (group 3); and craniotomy (group 4). The recurrence rate of $2.6 \%$ in group 1 was significantly lower than those in the other groups: $29.4 \%$ in group 2 , $39.5 \%$ in group 3 , and $44.4 \%$ in group 4 . Continuous irrigation did not result in additional complications.

Few studies have considered the use of intraoperative irrigation in twist drill craniostomy. Aoki found a significant reduction in the recurrence rate (from $29.2 \%$ to $6.7 \%$ ) using intraoperative irrigation in twist drill craniostomy (class III evidence). The use of irrigation had no impact on morbidity or mortality. ${ }^{12}$

\section{Drainage}

Four papers on the use of drainage met the criteria of class II evidence. Markwalder and Seiler ${ }^{31}$ discontinued a prospective study on burr hole craniostomy without closed system drainage: the results did not show significant differences in final outcome compared with Markwalder's previous study using a drain, ${ }^{9}$ but patients without drainage fared worse early after surgery. Wakai et al had significantly fewer recurrences with drainage (5\% v 33\%). ${ }^{4}$ In another study, the influence of the catheter position on the recurrence rate after burr hole craniostomy was analysed. Results were better when the tip of the drain was in a frontal position (5\% recurrences) than in a temporal (33\% recurrences), occipital (36\% recurrences), or parietal position ( $38 \%$ recurrences). ${ }^{35}$ Recently, Kwon et al correlated postoperative drainage volume with the recurrence rate. ${ }^{30}$ When the total drainage volume was below $200 \mathrm{ml}$, the recurrence rate increased from $0 \%$ to $6.4 \%$. This difference was highly significant. The use of a drain did not alter morbidity, mortality, or the cure rate in any of the publications reviewed.

We did not find any study comparing twist drill craniostomy with and without drainage. Figure 2 summarises the accumulated data from six publications that provided complete statistical data on 451 patients treated by twist drill craniostomy.

\section{Treatment of recurrences}

Twenty publications contained detailed data on the treatment of recurrences after burr hole craniostomy in 229 patients. $^{49151718222327-2931384043464752-55}$ One hundred and ninety four patients $(85 \%)$ were successfully treated by the 

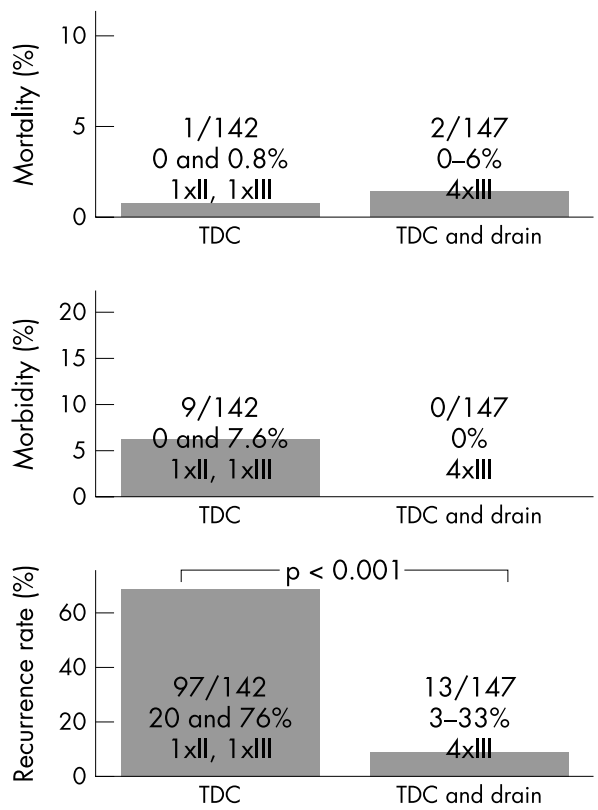

Figure 2 The effect of a drain in twist drill craniostomy. Comparison of mortality, morbidity, and recurrence rate for simple twist drill craniostomy (TDC) and TDC with drain. The grey columns show the relative percentage of summarised data on corresponding treatment groups from different publications. The absolute numbers are shown in the legend within the columns. In addition, the range of relative values and the number of studies which provided statistical data are listed with their classes of evidence.

same procedure as previously, $32(14 \%)$ underwent craniotomy, and three (1\%) died.

Data on 151 patients who suffered recurrences after twist drill craniostomy were available from seven publications. $^{6}{ }^{12} 1316414446$ One hundred and six patients (70\%) were successfully treated by the same approach in a second or third operation, while in 35 patients $(24 \%)$, burr hole craniostomy was considered more adequate for reoperation, and in 10 $(6 \%)$ craniotomy was thought to be the most useful procedure.

No data were available on treatment of recurrences after craniotomy.

Odds ratios of the summary data

Analysis of the odds ratios and 95\% confidence intervals of the summary data presented in fig 1 and 2 is shown in table 3 . The results of this analysis specify the relative risks of the different therapeutic techniques and options.

\section{Evidentiary evaluation of results}

\section{Surgical approach}

Type $A$ or $B$ recommendations: evaluation of the data does not allow any type A or B recommendations.

Type $\mathrm{C}$ recommendations

- Twist drill craniostomy and burr hole craniostomy are the safest procedures.
- Burr hole craniostomy and craniotomy are the most effective procedures.

- Burr hole craniostomy has the best cure to complication ratio.

\section{Irrigation}

Type A or B recommendations

Evaluation of data does not allow any type A or B recommendations.

Type $C$ recommendations

- Irrigation lowers the risk of recurrence in twist drill craniostomy.

- Irrigation does not increase the risk of infection.

\section{Drainage}

Type A recommendations

Evaluation of data does not allow any type A recommendations.

Type $B$ recommendations

- Drainage reduces the risk of recurrence in burr hole craniostomy.

- Frontal position of the drain reduces the risk of recurrence.

Type $C$ recommendations

- Drainage reduces the risk of recurrence in twist drill craniostomy.

- The use of a drain does not increase the risk of infection.

Treatment of recurrences

Type $A$ or $B$ recommendations

Evaluation of data does not allow any type A or B recommendations.

\section{Type $C$ recommendations}

- Burr hole craniostomy is more effective in treating recurrent haematoma than twist drill craniostomy.

- Craniotomy should be considered as the treatment of last choice.

\section{DISCUSSION}

The conclusions of our analysis differ from those of Markwalder's comprehensive review published in 1981 in several ways. $^{2}$ At that time, neurosurgical opinion on the treatment of chronic subdural haematoma was still influenced by the previous concept that this was a possibly lethal disorder. A large craniotomy with capsulectomy was a common operation..$^{5758}$ Markwalder's review on chronic subdural haematoma was an important step in minimising the invasiveness of the surgical treatment. The publications cited in our present review give clear evidence of this development. Nevertheless, the extent of surgery necessary for adequate treatment of chronic subdural haematoma is still a matter of debate. Up to now there have been no prospective randomised studies to determine which surgical approach is most appropriate. As has been shown,

\begin{tabular}{|c|c|c|c|c|}
\hline & Mortality & Morbidity & Cure rate & Recurrence rate \\
\hline TDC $\vee$ BHC & $1 \quad(0.6$ to 1.9$)$ & 0.8 (0.4 to 1.5$)$ & 2.0 (1.4 to 2.8 ) & 3.6 (2.8 to 4.5$)$ \\
\hline Craniotomy v BHC & $1.7(0.7$ to 4.0$)$ & 3.5 (1.9 to 6.4$)$ & 0.6 (0.4 to 0.8$)$ & $0.9(0.5$ to 1.5$)$ \\
\hline Craniotomy v TDC & $1.6(0.6$ to 4.5$)$ & 4.6 (1.9 to 10.9$)$ & $0.3(0.2$ to 0.5$)$ & 0.2 (0.1 to 0.4$)$ \\
\hline$T D C+$ drain $v$ TDC & $1.9(0.2$ to 21.7$)$ & $0.9(0.9 \text { to } 1.0)^{\prime}$ & - & $0.05(0.02$ to 0.09$)$ \\
\hline
\end{tabular}


contemporary treatment options range from simple twist drill craniostomy without drainage to large craniotomies with marsupialisation of haematoma membranes. ${ }^{19}{ }^{41}$

Burr hole craniostomy seems to have been the most commonly performed procedure for decompressing chronic subdural haematomas within the past 20 years (table 2 ). The widespread use of the burr hole technique is supported and justified by its safety. Morbidity and mortality rates of burr hole craniostomy are comparable with those of twist drill craniostomy, while the recurrence rates are similar to those achieved with craniotomy. Craniotomy is still the surgical approach with the least risk of recurrence. The downside of this technique, however, is that it has the greatest number of treatment related deaths, and it is also associated with the highest morbidity. It is conceivable that selection bias may have resulted in a somewhat distorted picture of the mortality and morbidity of craniotomy, as series of primary craniotomies for the treatment of this condition in general include patients who were presumed to have a higher risk of recurrence or who had a less satisfactory preoperative performance. $^{38394350}$ Thus it can be concluded that the burr hole technique shares the advantages of twist drill craniostomy, with its high cure rate and low risk of morbidity and mortality, and of primary craniotomy, with its low risk of recurrence. The greater number of publications on burr hole craniostomy over the years probably reflects the fact that most neurosurgeons now favour this approach. One argument in favour of the less invasive twist drill craniostomy is the positive correlation between the degree of invasiveness and the number of non-surgical complications in elderly people. ${ }^{3}$ Although theoretically attractive, this issue needs further study because of the paucity of data.

When we consider recurrence rate as a measure of the efficacy of treatment for the underlying process, a large decompression with its low recurrence rate appears to be the most rational approach. The recurrence rate of burr hole craniostomy, however, comes close to that of craniotomy. Whereas it is the goal of craniotomy, in general, to remove the haematoma and its membranes, burr hole craniostomy merely aims to release the haematoma fluid. In other words, removal of the haematoma membranes does not necessarily decrease the risk of recurrence. Therefore, it may be speculated that the haematoma itself is the promoter for its chronicity, and removal of the haematoma fluid should suffice as the primary goal of surgery. This hypothesis is supported by recent laboratory studies showing that the haematoma fluid contains large concentrations of vasoactive cytokines. ${ }^{59}{ }^{60}$ The high concentration of vascular endothelium derived growth factor (VEGF), for example, could be involved in the formation of the haematoma membranes. Furthermore, the haematoma fluid contains inflammatory mediators ${ }^{61-63}$ and fibrinolytic factors $^{6476}$ which all may play a role in the further development of the haematoma. The risk of recurrence is increased when the concentration of fibrinolytic factors remains high in the postoperative drainage fluid. ${ }^{74}$ The minor importance of membrane removal is further supported by clinical experience with endoscopy aided decompression of recurrent haematomas without excision of their membranes. ${ }^{23}$ 77-79

The intention of irrigation is to remove the haematoma completely or at least to dilute its contents. Clinical data support the beneficial effect of intraoperative irrigation when used in conjunction with twist drill craniostomy. As summarised above, the evidence for the benefit of irrigation in burr hole craniostomy is less strong. Continuous postoperative irrigation may be useful to reduce recurrences. The available data do not support the assumption that the use of irrigation increases the risk of infection. ${ }^{46}$

Recurrences may be treated by repeated twist drill craniostomy or by burr hole craniostomy. Here again, burr hole craniostomy appears to be superior to twist drill craniostomy, con-

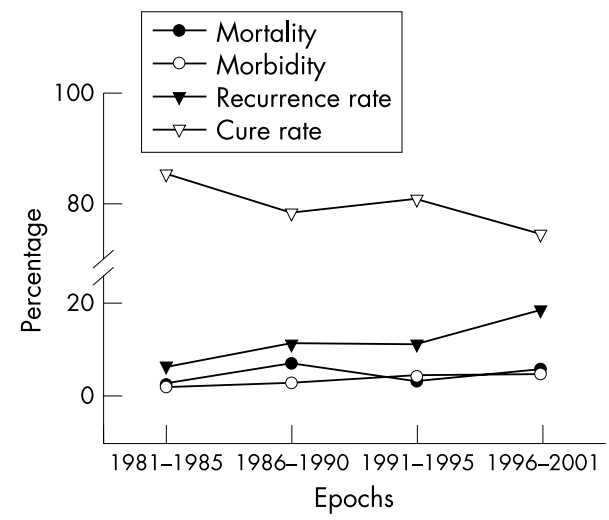

Figure 3 Cumulative summary of data on treatment of chronic subdural haematoma within epochs of five years. The analysis shows stable rates for mortality, morbidity, and cure rate as expressed by summarised mean values from papers published between 1981 and 2001.

sidering that about one third of recurrent haematomas were thought not to be suited for repeat twist drill craniostomy, while only one seventh of recurrent haematomas in patients who underwent burr hole craniostomy needed more invasive surgery. ${ }^{4} 6915-1820222327-293133384043464752-55$

The most convincing data on the treatment of chronic subdural haematoma refer to the use of a drain in burr hole craniostomy. The four class II evidence publications highlight different aspects of drainage systems. Notably, these data allow the only type B recommendations for chronic subdural haematoma surgery. One of the most remarkable findings is that low drainage volumes increase the risk of recurrence of the haematoma. ${ }^{30}$ Drainage appears also to reduce the risk of recurrence in conjunction with twist drill craniostomy.

Chronic subdural haematoma develops over a period of more than two weeks. ${ }^{80}$ During this period, about $90 \mathrm{ml}$ of haematoma fluid on average have to be buffered by the cranial reserve capacity without a detrimental rise in intracranial pressure. ${ }^{67}$ Postoperative drainage for several days might be useful to support re-expansion of the brain into the space which had been occupied by the haematoma. Nakaguchi and colleagues thought that the lower risk of recurrence when placing the tip of a drain in the frontal convexity was due to the removal of subdural air. ${ }^{35}$ Subdural air had earlier been identified as a risk factor of postoperative recurrence. ${ }^{81}$ It remains unclear, however, whether subdural air is the cause of postoperative recurrence or whether it is a sign indicating inadequate expansion of the brain.

We think that our analysis presents a representative review of the contemporary standards of treatment of chronic subdural haematoma. As with other analyses on topics such as unruptured aneurysms ${ }^{82}$ or Parkinson's disease, ${ }^{7}$ however, there are several methodological problems inherent in such types of systematic review. Publication bias is related to the arbitrary selection of language and by primarily selecting manuscripts from a database such as Medline, yielding papers that are more likely to report on positive results. Furthermore, the lack of any studies providing class I evidence and the paucity of studies providing class II evidence produces lower quality data and precludes the application of statistical procedures for meta-analysis.

The presently available data do not allow us to recommend "standard treatments" or "guidelines" for chronic subdural haematoma surgery. Furthermore, there is no information so far on whether one approach is superior to another with regard to the stage of chronicity of the haematoma. It is obvious that the least invasive treatment option-that is, twist drill craniostomy without irrigation or postoperative drainage_can provide a cure in certain patients, while others 
need a more invasive approach such as craniotomy. It will be a task for the future to identify predictive factors that will enable one to tailor the surgical approach for the individual patient. Finally, a cumulative summary of data on the treatment of chronic subdural haematoma published within epochs of five years suggests that variations in the principal surgical techniques have not substantially improved the outcome over the past 20 years (fig 3). There is a need for properly conducted prospective trials on surgery for chronic subdural haematoma. It might also be worthwhile seeking alternative strategies in the treatment of this condition.

\section{Conclusions}

This evidence based review of contemporary surgical techniques for the treatment of chronic subdural haematoma identified twist drill craniostomy and burr hole craniostomy as the safest methods. Burr hole craniostomy has the best cure to complication ratio and is superior to twist drill craniostomy in the treatment of recurrences. Craniotomy and burr hole craniostomy have the lowest recurrence rates. The use of closed system drainage reduces the risk of recurrence without additional risk of complications.

\section{Authors' affiliations}

R Weigel, P Schmiedek, J K Krauss, Department of Neurosurgery,

University Hospital, Klinikum Mannheim, Mannheim, Germany

\section{Competing interests: none declared}

\section{REFERENCES}

1 Virchow R. Das Hämatom der Dura mater. Verh Phys Med Ges Würzburg 1857;7:134-42

2 Markwalder TM. Chronic subdural hematomas: a review. J Neurosurg $1981 ; 54: 637-45$.

3 Rohde V, Graf G, Hassler W. Complications of burr-hole craniostomy and closed-system drainage for chronic subdural hematomas: a retrospective analysis of 376 patients. Neurosurg Rev 2001;69:40-7.

4 Wakai S, Hashimoto K, Watanabe N, et al. Efficacy of closed-system drainage in treating chronic subdural hematoma: a prospective comparative study. Neurosurgery 1990;26:771-3.

5 Beatty RA. Subdural haematomas in the elderly: experience with treatment by trephine craniotomy and not closing the dura or replacing the bone plate. Br J Neurosurg 1999;13:60-4.

6 Camel M, Grubb RL. Treatment of chronic subdural hematoma by twist-drill craniotomy with continuous catheter drainage. J Neurosurg 1986;65:183-7

7 Hallett M, Litvan I. Evaluation of surgery for Parkinson's disease: a report of the therapeutics and technology assessment subcommittee of the American Academy of Neurology. The Task Force on Surgery for Parkinson's Disease. Neurology 1999;53:1910-21.

8 Guidelines for the management of severe head injury. Brain Trauma Foundation, American Association of Neurological Surgeons, Joint Section on Neurotrauma and Critical Care. J Neurotrauma 1996;13:641-734

9 Markwalder TM, Steinsiepe KF, Rohner M, et al. The course of chronic subdural hematomas after burr-hole craniostomy and closed-system drainage. J Neurosurg 1981;55:390-6.

10 Bender MB, Christoff N. Nonsurgical treatment of subdural hematomas. Arch Neurol 1974;31:73-9.

11 Jennett B, Bond $M$. Assessment of outcome after severe brain damage. Lancet 1975;i:480-4.

12 Aoki N. Subdural tapping and irrigation for the treatment of chronic subdural hematoma in adults. Neurosurgery 1984;14:545-8

13 Aoki N. A new therapeutic method for chronic subdural hematoma in adults: replacement of the hematoma with oxygen via percutaneous subdural tapping. Surg Neurol 1992;38:253-6.

14 Aydin IH, Aydin Y, Akdemir D, et al. Chronic subdural hematomas (clinical analysis). Zentralb/ Neurochir 1987;48:308-1 1

15 Benzel EC, Bridges RM, Hadden TA, et al. The single burr hole technique for the evacuation of non-acute subdural hematomas. J Trauma 1994:36:190-4.

16 Carlton CK, Saunders RL. Twist drill craniostomy and closed system drainage of chronic and subacute subdural hematomas. Neurosurgery 1983; 13:153-9.

17 Choudhury AR. Avoidable factors that contribute to complications in the surgical treatment of chronic subdural haematoma. Acta Neurochir 1994;129:15-19.

18 Drapkin AJ. Chronic subdural hematoma: pathophysiological basis for treatment. Br J Neurosurg 1991;5:467-73.

19 Ernestus RI, Beldzinski $\mathrm{P}$, Lanfermann $\mathrm{H}$, et al. Chronic subdural hematoma: surgical treatment and outcome in 104 patients. Surg Neurol 1997;48:220-5.
20 Grisoli F Graziani N, Peragut JC, et al. Perioperative lumbar injection of Ringer's lactate solution in chronic subdural hematomas: a series of 100 cases. Neurosurgery 1988;23:616-21.

21 Hamilton MG, Frizzell JB, Tranmer BI. Chronic subdural hematoma: the role for craniotomy re-evaluated. Neurosurgery 1993;33:67-72.

22 Harders A, Eggert HR, Weigel K. Treatment of chronic subdural haematoma by closed external drainage. Neurochirurgia 1982;25:147-52.

23 Hellwig D, Kuhn TJ, Bauer BL, et al. Endoscopic treatment of septated chronic subdural hematoma. Surg Neurol 1996:45:272-7.

24 Hennig R, Kloster R. Burr hole evacuation of chronic subdural haematomas followed by continuous inflow and oufflow irrigation. Acta Neurochir 1999;141:171-6

25 Iwabuchi T, Sekiya T, Suzuki S. Neurosurgical aspects of the parietal boss in patients with chronic subdural hematomas. Neurosurgery $1981 ; 9: 531-4$

26 Kitakami A, Ogawa A, Hakozaki S, et al. Carbon dioxide gas replacement of chronic subdural hematoma using single burr-hole irrigation. Surg Neurol 1995;43:574-7.

27 Kotwica Z, Brzezinski J. Chronic subdural haematoma treated by burr holes and closed system drainage: personal experience in 131 patients. Br J Neurosurg 1991;5:461-5.

28 Krupp WF, Jans PJ. Treatment of chronic subdural haematoma with burr-hole craniostomy and closed drainage. Br J Neurosurg 1995;9:619-27.

29 Kuroki T, Matsumoto M, Kushida T, et al. Nontraumatic subdural hematoma secondary to dural metastasis of lung cancer: case report and review of the literature. No Shinkei Geka 1994;22:857-62.

30 Kwon TH, Park YK, Lim DJ, et al. Chronic subdural hematoma: evaluation of the clinical significance of postoperative drainage volume. J Neurosurg 2000;93:796-9

31 Markwalder TM, Seiler RW. Chronic subdural hematomas: to drain or not to drain? Neurosurgery 1985;16:185-8

32 Matsumoto K, Akagi K, Abekura M, et al. Recurrence factors for chronic subdural hematomas after burr-hole craniostomy and closed system drainage. Neurol Res 1999;21:277-80.

33 Mellergard $\mathbf{P}$, Wisten $\mathrm{O}$. Operations and re-operations for chronic subdural haematomas during a 25 -year period in a well defined population. Acta Neurochir 1996;138:708-13.

34 Merlicco G, Pierangeli E, di Padova PL. Chronic subdural hematomas in adults: prognostic factors. Analysis of 70 cases. Neurosurg Rev 1995; 18:247-51.

35 Nakaguchi H, Tanishima T, Yoshimasu N. Relationship between drainage catheter location and postoperative recurrence of chronic subdural hematoma after burr-hole irrigation and closed-system drainage. J Neurosurg 2000:93:791-5.

36 Ohaegbulam SC. Surgically treated traumatic subacute and chronic subdural haematomas: a review of 132 cases. Injury 1981;13:23-6.

37 Patrick D, Gates PC. Chronic subdural haematoma in the elderly. Age Ageing 1984;13:367-9

38 Piotrowski WP, Krombholz-Reindl MA. Surgical outcome in chronic subdural hematoma. Unfallchirurgie 1996;22:110-16.

39 Probst C. Peritoneal drainage of chronic subdural hematomas in older patients. J Neurosurg 1988;68:908-11

$40 \operatorname{Ram} Z$, Hadani $M$, Sahar $A$, et al. Continuous irrigation-drainage of the subdural space for the treatment of chronic subdural haematoma. A prospective clinical trial. Acta Neurochir 1993;120:40-3.

41 Reinges MH, Hasselberg I, Rohde V, et al. Prospective analysis of bedside percutaneous subdural tapping for the treatment of chronic subdural haematoma in adults. J Neurol Neurosurg Psychiatry 2000;69:40-7

42 Richter HP, Klein HJ, Schafer M. Chronic subdural haematomas treated by enlarged burr-hole craniotomy and closed system drainage. Retrospective study of 120 patients. Acta Neurochir 1984;71:179-88.

43 Robinson RG. Chronic subdural hematoma: surgical management in 133 patients. J Neurosurg 1984:61:263-8.

44 Rychlicki F, Recchioni MA, Burchianti M, et al. Percutaneous twist-drill craniostomy for the treatment of chronic subdural haematoma. Acta Neurochir 1991;113:38-41.

45 Schulz W, Saballus R, Flugel R, et al. Chronic subdural hematoma. A comparison of bore hole trepanation and craniotomy. Zentralb Neurochir 1988:49:280-9.

46 Smely C, Madlinger A, Scheremet R. Chronic subdural haematoma-a comparison of two different treatment modalities. Acta Neurochir 1997; 139:818-25.

47 Spallone A, Giuffre R, Gagliardi FM, et al. Chronic subdural hematoma in extremely aged patients. Eur Neurol 1989;29:18-22.

48 Stroobandt G, Fransen P, Thauvoy C, et al. Pathogenetic factors in chronic subdural haematoma and causes of recurrence after drainage. Acta Neurochir 1995;137:6-14.

49 Suzuki K, Sugita K, Akai T, et al. Treatment of chronic subdural hematoma by closed-system drainage without irrigation. Surg Neurol 1998;50:231-4

50 Tanikawa M, Mase M, Yamada K, et al. Surgical treatment of chronic subdural hematoma based on intrahematomal membrane structure on MRI. Acta Neurochir 2001:143:613-18.

51 Tsutsumi K, Maeda K, lijima A, et al. The relationship of preoperative magnetic resonance imaging findings and closed system drainage in the recurrence of chronic subdural hematoma. J Neurosurg 1997;87:870-5.

52 van Havenbergh T, van Calenbergh F, Goffin J, et al. Outcome of chronic subdural haematoma: analysis of prognostic factors. $\mathrm{Br} J$ Neurosurg 1996;10:35-9. 
53 Weir BK. Results of burr hole and open or closed suction drainage for chronic subdural hematomas in adults. Can J Neurol Sci 1983;10:22-6. 54 Weisse A, Berney J. Chronic subdural haematomas. Results of a closed drainage method in adults. Acta Neurochir 1994;127:37-40.

55 Yoshimoto Y, Kwak S. Frontal small craniostomy and irrigation for treatment of chronic subdural haematoma. $\mathrm{Br} J$ Neurosurg 1997;11:150-1.

56 Kuroki T, Katsume $M$, Harada $N$, et al. Strict closed-system drainage for treating chronic subdural haematoma. Acta Neurochir 2001;143:1041-4.

57 Robinson RG. The treatment of subacute and chronic subdural hematomas. BM 1955;1:21-2.

58 Sherman IJ, Fromm SM. Subdural hematoma. Conn Med 1961;25:569-73.

59 Suzuki K, Takano S, Nose T, et al. Increased concentration of vascular endothelial growth factor (VEGF) in chronic subdural hematoma. $J$ Trauma 1999;46:532-3

60 Weigel R, Schilling L, Schmiedek P. Specific pattern of growth factor distribution in chronic subdural hematoma (CSH): evidence for an angiogenic disease. Acta Neurochir 2001;143:811-18.

61 Fujisawa $\mathbf{H}$, Ito $\mathrm{H}$, Kashiwagi $\mathrm{S}$, et al. Kallikrein-kinin system in chronic subdural haematomas: its roles in vascular permeability and regulation of fibrinolysis and coagulation. J Neurol Neurosurg Psychiatry 1995:59:388-94.

62 Hirashima Y, Nagahori T, Nishijima M, et al. Analysis of plasma and hematoma lipids related to choline glycerophospholipid in patients with chronic subdural hematoma. Neurol Med Chir 1994;34:131-5.

63 Matsumori K, Yoshioka M. Kinetics of prostaglandin and its significance in chronic subdural hematoma. Neurol Med Chir 1987;27:498-504.

64 Ito $\mathbf{H}$, Komai T, Yamamoto S. Fibrin and fibrinogen degradation products in chronic subdural hematoma. Neurol Med Chir (Tokyo) $1975 ; 15: 51-5$

65 Ito $\mathbf{H}$, Yamamoto S, Komai T, et al. Role of local hyperfibrinolysis in the etiology of chronic subdural hematoma. I Neurosurg 1976:45:26-31.

66 Ito H, Komai T, Yamamoto S. Fibrinolytic enzyme in the lining walls of chronic subdural hematoma. J Neurosurg 1978;48:197-200.

67 Kawakami Y, Chikama M, Tamiya T, et al. Coagulation and fibrinolysis in chronic subdural hematoma. Neurosurgery 1989;25:25-9.

68 Kawakami Y, Tanimoto T, Shimamura Y. Coagulopathy in chronic subdural hematoma. Neurol Med Chir 1991;31:32-6.
69 Komai T, Ito H, Yamashima T, et al. Etiology of chronic subdural hematoma-role of local hyperfibrinolysis. Neurol Med Chir 1977; 17:499-505.

70 Labadie EL, Glover D. Chronic subdural hematoma: concepts of physiopathogenesis. A review. Can J Neurol Sci 1974;1:222-5.

71 Lim DJ, Chung YG, Park YK, et al. Relationship between tissue plasminogen activator, plasminogen activator inhibitor and CT image in chronic subdural hematoma. J Korean Med Sci 1995;10:373-8.

72 Nomura S, Kashiwagi S, Ito H, et al. Degradation of fibrinogen and fibrin by plasmin and nonplasmin proteases in the chronic subdural hematoma: evaluation by sodium dodecyl sulfate-polyacrylamide gel electrophoresis and immunoblot. Electrophoresis 1993;14:1318-21.

73 Nomura S Kashiwagi S, Fujisawa $\mathrm{H}$, et al. Characterization of local hyperfibrinolysis in chronic subdural hematomas by SDS-PAGE and immunoblot. J Neurosurg 1994;81:910-13.

74 Saito $\mathbf{K}$, Ito $\mathrm{H}$, Hasegawa $T$, et al. Plasmin-alpha 2-plasmin inhibitor complex and alpha 2-plasmin inhibitor in chronic subdural hematoma. J Neurosurg 1989;70:68-72.

75 Toyosawa M, Kashiwagi S, Pei W, et al. Electrophoretic demonstration of high molecular weight fibrin degradation products persisting in chronic subdural hematomas. Electrophoresis 1997;18:118-21.

76 Weir B, Gordon P. Factors affecting coagulation: fibrinolysis in chronic subdural fluid collections. J Neurosurg 1983;58:242-5

77 Drapkin AJ. Endoscopy for chronic SDH. Surg Neurol 1997;48:427.

78 Gruber DP, Crone KR. Endoscopic washout: a new technique for treating chronic subdural hematomas in infants. Pediatr Neurosurg 1997;27:292-5.

79 Rodziewicz GS, Chuang WC. Endoscopic removal of organized chronic subdural hematoma. Surg Neurol 1995;43:569-72.

80 McKissock W, Richardson A, Bloom WH. Subdural hematoma. A review of 389 cases. Lancet 1960;i:1365-9.

81 Nagata K, Asano T, Basugi N, et al. Studies on the operative factors affecting the reduction of chronic subdural hematoma, with special reference to the residual air in the hematoma cavity. No Shinkei Geka 1989:17:15-20.

82 Brennan JW, Schwartz ML. Unruptured intracranial aneurysms: appraisal of the literature and suggested recommendations for surgery, using evidence-based medicine criteria. Neurosurgery 2000;47:1359-71.

\section{$\mathrm{BM}$}

\section{3rd Asia Pacific Forum on Quality Improvement in Health Care} 3-5 September 2003, Auckland, New Zealand

We are delighted to announce this forthcoming conference in Auckland, New Zealand.

The themes of the 3rd Asia Pacific Forum on Quality Improvement in Health Care are:

- Agenda for quality: Improving equity in health care delivery

- Improving safety

- Leadership for improvement

- Measuring quality and benchmarking for change

- Evidence based knowledge and education for quality improvement

- Improving health systems

- Patient/consumer centred quality improvement

Presented to you by the BM Publishing Group (London, UK) and Institute for Healthcare Improvement (Boston, USA), supported by the New Zealand Ministry of Health, ACC, and Standards New Zealand.

For more information about the Forum or to register contact: quality@bma.org.uk or go to:

www.quality.bmipg.com

Tel: +44 (0)2073836409 Fax: +44 (0)207383 6869 